\title{
BOUNDS ON TOEPLITZ DETERMINANT FOR STARLIKE FUNCTIONS WITH RESPECT TO CONJUGATE POINTS
}

\section{DAUD MOHAMAD, NUR HAZWANI AQILAH ABDUL WAHID*}

Faculty of Computer and Mathematical Sciences, Universiti Teknologi MARA, 40450 Shah Alam, Selangor, Malaysia

*Corresponding author: hazwaniaqilah@tmsk.uitm.edu.my

ABSTRACT. This paper is concerned with the estimate of the upper bounds of the Toeplitz determinants $\left|T_{2}(3)\right|$ and $\left|T_{3}(3)\right|$ for functions belonging to the subclass of starlike functions with respect to conjugate points. The results presented would extend the results for some existing subclasses in the literature.

\section{INTRODUCTION}

Let $\mathrm{A}$ be the class of functions $f(z)$ which are analytic in an open unit disk $E=\{z:|z|<1\}$ and having the power series expansion

$$
f(z)=z+\sum_{n=2}^{\infty} a_{n} z^{n}
$$

in $E$. Let $S$ be the class of functions $f(z) \in \mathrm{A}$ and univalent in $E$.

Let $P$ be the class of functions $p(z)$ of the form

Received February 28th, 2021; accepted April 7th, 2021; published May 4th, 2021.

2010 Mathematics Subject Classification. 30C45, 30C50.

Key words and phrases. coefficient bounds; univalent functions; starlike functions; Toeplitz determinants

(C)2021 Authors retain the copyrights of their papers, and all open access articles are distributed under the terms of the Creative Commons Attribution License. 


$$
p(z)=1+\sum_{n=1}^{\infty} p_{n} z^{n}
$$

that is analytic in $E$ and satisfying the condition $\operatorname{Re} p(z)>0, z \in E$. Functions in $P$ are called Carathéodory functions. It is well known that if $p(z) \in P$, then a Schwarz function $\omega(z)$ exists with $\omega(0)=0,|\omega(z)|<1, z \in E$ such that [1]

$$
p(z)=\frac{1+\omega(z)}{1-\omega(z)}
$$

For two functions $F(z)$ and $G(z)$ analytic in $E$, we say that the function $F(z)$ is subordinate to $G(z)$ and we write it as $F(z) \prec G(z)$ if there exists a Schwarz function $\omega(z)$ which is analytic in $E$ with $\omega(0)=0,|\omega(z)|<1$, such that $F(z)=G(\omega(z))$. Further, if $G(z)$ is univalent in $E$, then $F(z) \prec G(z) \Leftrightarrow F(0)=G(0)$ and $F(E)=G(E)$ (see Miller and Mocanu [2, 3] for details).

Let $S^{*}$ denote the class of starlike functions in $S$. It is known that $f(z) \in S^{*}$ if and only if

$$
\operatorname{Re}\left\{\frac{z f^{\prime}(z)}{f(z)}\right\}>0, z \in E .
$$

El-Ashwah and Thomas [4] defined the following class:

$$
S_{C}{ }^{*}=\left\{f(z) \in \mathrm{A}: \operatorname{Re}\left\{\frac{2 z f^{\prime}(z)}{f(z)+\overline{f(\bar{z})}}\right\}>0, z \in E\right\} .
$$

Functions in the class $S_{C}{ }^{*}$ are called starlike functions with respect to conjugate points.

Halim [5] defined the following class:

$$
S_{C}{ }^{*}(\delta)=\left\{f(z) \in \mathrm{A}: \operatorname{Re}\left\{\frac{2 z f^{\prime}(z)}{f(z)+\overline{f(\bar{z})}}\right\}>\delta, 0 \leq \delta<1, z \in E\right\} .
$$

In terms of subordination, Dahhar and Janteng [6] generalized the class $S_{C}{ }^{*}$ and it is denoted by $S_{C}{ }^{*}(A, B)$. This class is defined as follows: 


$$
S_{C}^{*}(A, B)=\left\{f(z) \in \mathrm{A}: \frac{2 z f^{\prime}(z)}{f(z)+\overline{f(\bar{z})}} \prec \frac{1+A z}{1+B z},-1 \leq B<A \leq 1, z \in E\right\} .
$$

Wahid et al. [7] introduced the subclass of tilted starlike functions with respect to conjugate points of order $\delta, S_{C}^{*}(\alpha, \delta, A, B)$ and it is given by

$$
S_{C}{ }^{*}(\alpha, \delta, A, B)=\left\{f(z) \in \mathrm{A}:\left[e^{i \alpha} \frac{z f^{\prime}(z)}{g(z)}-\delta-i \sin \alpha\right] \frac{1}{t_{\alpha \delta}} \prec \frac{1+A z}{1+B z}, z \in E\right\},
$$

where $g(z)=\frac{f(z)+\overline{f(\bar{z})}}{2}, t_{\alpha \delta}=\cos \alpha-\delta>0,0 \leq \delta<1,|\alpha|<\frac{\pi}{2}$ and $-1 \leq B<A \leq 1$.

In particular, $S_{C}{ }^{*}(0) \equiv S_{C}{ }^{*}, S_{C}^{*}(1,-1) \equiv S_{C}{ }^{*}$ and $S_{C}{ }^{*}(0,0,1,-1) \equiv S_{C}{ }^{*}$.

Toeplitz matrices are one of the well-studied classes of structured matrices. The concept of Toeplitz matrices led to the development of the studies related to Toeplitz determinants, Toeplitz kernel, Toeplitz operators, and q-deformed Toeplitz matrices [8]. In a recent investigation, the Toeplitz determinant has been studied by [9-18], and they succeeded in estimating the coefficient bounds for Toeplitz determinant $\left|T_{q}(n)\right|, n, q \geq 1$ for the first few values of $n$ and $q$ over some subclasses of A. The Toeplitz determinant $T_{q}(n), n, q \geq 1$ of functions $f(z)$ of the form (1.1), is defined by Thomas and Halim [9]

$$
T_{q}(n)=\left|\begin{array}{cccc}
a_{n} & a_{n+1} & \cdots & a_{n+q-1} \\
a_{n+1} & a_{n} & \cdots & a_{n+q-2} \\
\vdots & \vdots & \vdots & \vdots \\
a_{n+q-1} & a_{n+q-2} & \cdots & a_{n}
\end{array}\right|, a_{1}=1 .
$$

However, apart from these works, there was no study of finding estimates for $\left|T_{2}(3)\right|$ and $\left|T_{3}(3)\right|$ for the subclasses introduced by El-Ashwah and Thomas [4], Halim [5], Dahhar and Janteng [6], and Wahid et al. [7]. In fact, as far as we are concerned, no bound for $\left|T_{3}(3)\right|$ was obtained for the class of univalent functions and its subclasses in the existing literature. Therefore, in this paper, we obtain the upper bounds for the Toeplitz determinant for $S_{C}{ }^{*}(\alpha, \beta, A, B)$ as defined in (1.7) for the case of $n=3, q=2$ and $n=3, q=3$ namely 


$$
T_{2}(3)=\left|\begin{array}{ll}
a_{3} & a_{4} \\
a_{4} & a_{3}
\end{array}\right|
$$

and

$$
T_{3}(3)=\left|\begin{array}{lll}
a_{3} & a_{4} & a_{5} \\
a_{4} & a_{3} & a_{4} \\
a_{5} & a_{4} & a_{3}
\end{array}\right| .
$$

We also give some results for the subclasses introduced by El-Ashwah and Thomas [4], Halim [5], and Dahhar and Janteng [6].

We shall state the following lemmas to prove our main results.

\section{PRELIMINARY RESULTS}

Lemma 2.1. [19] For a function $p(z) \in P$ of the form (1.2), the sharp inequality $\left|p_{n}\right| \leq 2$ holds for each $n \geq 1$. Equality holds for the function $p(z)=\frac{1+z}{1-z}$.

Lemma 2.2. [20] Let $p(z) \in P$ of the form (1.2) and $\mu \in \mathbb{C}$. Then

$$
\left|p_{n}-\mu p_{k} p_{n-k}\right| \leq 2 \max \{1,|2 \mu-1|\}, 1 \leq k \leq n-1 .
$$

If $|2 \mu-1| \geq 1$, then the inequality is sharp for the function $p(z)=\frac{1+z}{1-z}$ or its rotations. If $|2 \mu-1|<1$, then the inequality is sharp for the function $p(z)=\frac{1+z^{n}}{1-z^{n}}$ or its rotations.

\section{MAIN RESULTS}

Theorem 3.1. If the function $f(z)$ given by (1.1) belongs to the class $S_{C}^{*}(\alpha, \delta, A, B)$, then

$$
\begin{aligned}
\left|T_{2}(3)\right| \leq & \frac{T^{2}}{2304}\left\{832+64\left|(1-3 \Upsilon) \xi^{4}+\left(-3 \Upsilon+11 \Upsilon^{2}\right) \xi^{3}+\left(-12 \Upsilon^{3}+2 \Upsilon^{2}\right) \xi^{2}+4 \Upsilon^{4} \xi\right|\right. \\
& +8\left|72 \Upsilon^{2}-144 \Upsilon+72 \xi^{2}+(-144 \Upsilon+144) \xi\right|+8\left|-128 \Upsilon^{2}+128 \Upsilon-72 \xi^{2}+(192 \Upsilon-96) \xi\right| \\
& \left.+16\left|-16 \Upsilon^{2}+32 \Upsilon^{3}+\left(24 \Upsilon-88 \Upsilon^{2}+32 \Upsilon^{3}\right) \xi+(-24-12 \Upsilon) \xi^{3}+\left(-16+92 \Upsilon-72 \Upsilon^{2}\right) \xi^{2}\right|\right\}
\end{aligned}
$$

where $\xi=T e^{-i \alpha}, T=(A-B) t_{\alpha \delta}, t_{\alpha \delta}=\cos \alpha-\delta$ and $\Upsilon=1+B$. 
Proof. From (1.7), since $f(z) \in S_{C}{ }^{*}(\alpha, \delta, A, B)$, according to subordination relationship, so there exists a Schwarz function $\omega(z)$ such that

$$
\left\{e^{i \alpha} \frac{z f^{\prime}(z)}{g(z)}-\delta-i \sin \alpha\right\} \frac{1}{t_{\alpha \delta}}=\frac{1+A \omega(z)}{1+B \omega(z)},
$$

where $g(z)=\frac{f(z)+\overline{f(\bar{z})}}{2}, t_{\alpha \delta}=\cos \alpha-\delta$.

Define a function

$$
h(z)=\frac{1+\omega(z)}{1-\omega(z)}=1+\sum_{n=1}^{\infty} k_{n} z^{n}
$$

We have $h(z) \in P$ and

$$
\omega(z)=\frac{h(z)-1}{h(z)+1}
$$

Using (3.2), from (3.1), we have

$$
e^{i \alpha} \frac{z f^{\prime}(z)}{g(z)}=\frac{\left[e^{i \alpha}(1-B)-T\right]+h(z)\left[e^{i \alpha}(1+B)+T\right]}{1-B+h(z)(1+B)}
$$

where $T=(A-B) t_{\alpha \delta}$.

Using the series expansion in (3.3), we get

$$
\begin{aligned}
& e^{i \alpha}(1-B)\left(z+2 a_{2} z^{2}+3 a_{3} z^{3}+4 a_{4} z^{4}+\cdots\right) \\
& \quad+e^{i \alpha}(1+B)\left(z+2 a_{2} z^{2}+3 a_{3} z^{3}+4 a_{4} z^{4}+\cdots\right)\left(1+k_{1} z+k_{2} z^{2}+k_{3} z^{3}+\cdots\right) \\
& =\left[e^{i \alpha}(1-B)-T\right]\left(z+a_{2} z^{2}+a_{3} z^{3}+a_{4} z^{4}+\cdots\right) \\
& \quad+\left[e^{i \alpha}(1+B)+T\right]\left(z+a_{2} z^{2}+a_{3} z^{3}+a_{4} z^{4}+\cdots\right)\left(1+k_{1} z+k_{2} z^{2}+k_{3} z^{3}+\cdots\right) .
\end{aligned}
$$

Equating the coefficients of $z^{3}$ and $z^{4}$ respectively in the expansion of (3.4) and for simplicity, we take $\xi=T e^{-i \alpha}$ and $\Upsilon=1+B$, give us

$$
a_{3}=\frac{2 k_{2} \xi+k_{1}^{2} \xi^{2}-\Upsilon k_{1}^{2} \xi}{8}
$$


and

$$
a_{4}=\frac{8 k_{3} \xi+6 k_{1} k_{2} \xi^{2}-8 k_{1} k_{2} \Upsilon \xi+k_{1}{ }^{3} \xi^{3}-3 k_{1}{ }^{3} \Upsilon \xi^{2}+2 k_{1}{ }^{3} \Upsilon^{2} \xi}{48} .
$$

Squaring (3.5) and (3.6), respectively, we get

$$
a_{3}{ }^{2}=\frac{4 k_{2}{ }^{2} \xi^{2}+k_{1}{ }^{4} \Upsilon^{2} \xi^{2}-4 k_{1}{ }^{2} k_{2} \Upsilon \xi^{2}-2 k_{1}{ }^{4} \Upsilon \xi^{3}+4 k_{1}{ }^{2} k_{2} \xi^{3}+k_{1}{ }^{4} \xi^{4}}{64}
$$

and

$$
\begin{aligned}
a_{4}{ }^{2}= & \frac{\xi^{2}}{2304}\left[64 k_{3}^{2}+k_{1}{ }^{6} \xi^{4}-3 k_{1}^{6} \Upsilon \xi^{3}+2 k_{1}{ }^{6} \Upsilon^{2} \xi^{2}-3 k_{1}^{6} \Upsilon \xi^{4}+11 k_{1}^{6} \Upsilon^{2} \xi^{3}-12 k_{1}{ }^{6} \Upsilon^{3} \xi^{2}+4 k_{1}{ }^{6} \Upsilon^{4} \xi\right. \\
& +96 k_{1} k_{2} k_{3} \xi-128 \Upsilon k_{1} k_{2} k_{3}+16 k_{1}{ }^{3} k_{3} \xi^{2}-24 k_{1}{ }^{3} k_{3} \Upsilon \xi+16 \Upsilon^{2} k_{1}{ }^{3} k_{3}-24 k_{1}{ }^{3} k_{3} \Upsilon \xi^{2}+16 k_{1}{ }^{3} k_{3} \Upsilon^{2} \xi \\
& +36 k_{1}{ }^{2} k_{2}{ }^{2} \xi^{2}-96 k_{1}{ }^{2} k_{2}{ }^{2} \Upsilon \xi+64 \Upsilon^{2} k_{1}{ }^{2} k_{2}{ }^{2}+12 k_{1}{ }^{4} k_{2} \xi^{3}-34 k_{1}{ }^{4} k_{2} \Upsilon \xi^{2}+36 k_{1}{ }^{4} k_{2} \Upsilon^{2} \xi-16 \Upsilon^{3} k_{1}{ }^{4} k_{2} \\
& \left.+6 k_{1}{ }^{4} k_{2} \Upsilon \xi^{3}+36 k_{1}{ }^{4} k_{2} \Upsilon^{2} \xi^{2}-16 k_{1}{ }^{4} k_{2} \Upsilon^{3} \xi\right] .
\end{aligned}
$$

From the equations (1.8), (3.7), and (3.8), yield

$$
\begin{aligned}
\left|T_{2}(3)\right|= & \left|a_{4}{ }^{2}-a_{3}{ }^{2}\right| \\
= & \mid \frac{\xi^{2}}{2304}\left\{\left[64 k_{3}{ }^{2}+k_{1}{ }^{6} \xi^{4}-3 k_{1}{ }^{6} \Upsilon \xi^{3}+2 k_{1}{ }^{6} \Upsilon^{2} \xi^{2}-3 k_{1}{ }^{6} \Upsilon \xi^{4}+11 k_{1}^{6} \Upsilon^{2} \xi^{3}-12 k_{1}{ }^{6} \Upsilon^{3} \xi^{2}+4 k_{1}^{6} \Upsilon^{4} \xi\right.\right. \\
& +96 k_{1} k_{2} k_{3} \xi-128 \Upsilon k_{1} k_{2} k_{3}+16 k_{1}{ }^{3} k_{3} \xi^{2}-24 k_{1}{ }^{3} k_{3} \Upsilon \xi+16 \Upsilon^{2} k_{1}{ }^{3} k_{3}-24 k_{1}{ }^{3} k_{3} \Upsilon \xi^{2}+16 k_{1}{ }^{3} k_{3} \Upsilon^{2} \xi \\
& +36 k_{1}{ }^{2} k_{2}{ }^{2} \xi^{2}-96 k_{1}{ }^{2} k_{2}{ }^{2} \Upsilon \xi+64 \Upsilon^{2} k_{1}{ }^{2} k_{2}{ }^{2}+12 k_{1}{ }^{4} k_{2} \xi^{3}-34 k_{1}{ }^{4} k_{2} \Upsilon \xi^{2}+36 k_{1}{ }^{4} k_{2} \Upsilon^{2} \xi-16 \Upsilon^{3} k_{1}{ }^{4} k_{2} \\
& \left.+6 k_{1}{ }^{4} k_{2} \Upsilon \xi^{3}+36 k_{1}{ }^{4} k_{2} \Upsilon^{2} \xi^{2}-16 k_{1}{ }^{4} k_{2} \Upsilon^{3} \xi\right] \\
& \left.-\left[144 k_{2}{ }^{2}+36 \Upsilon^{2} k_{1}{ }^{4}-144 \Upsilon k_{1}{ }^{2} k_{2}-72 k_{1}{ }^{4} \Upsilon \xi+144 k_{1}{ }^{2} k_{2} \xi+36 k_{1}{ }^{4} \xi^{2}\right]\right\} \mid \\
& =\mid \frac{\xi^{2}}{2304}\left\{64 k_{3}{ }^{2}-144 k_{2}{ }^{2}+k_{1}^{6}\left[(1-3 \Upsilon) \xi^{4}+\left(-3 \Upsilon+11 \Upsilon^{2}\right) \xi^{3}+\left(2 \Upsilon^{2}-12 \Upsilon^{3}\right) \xi^{2}+4 \Upsilon^{4} \xi\right]\right. \\
& +k_{1}{ }^{4}\left[-36 \xi^{2}+72 \Upsilon \xi-36 \Upsilon^{2}\right]+k_{1}{ }^{2} k_{2}[-144 \xi+144 \Upsilon]+k_{1} k_{2} k_{3}[96 \xi-128 \Upsilon] \\
& +k_{1}{ }^{2} k_{2}{ }^{2}\left[36 \xi^{2}-96 \Upsilon \xi+64 \Upsilon^{2}\right]+k_{1}{ }^{3} k_{3}\left[16 \Upsilon^{2}+(16-24 \Upsilon) \xi^{2}+\left(-24 \Upsilon+16 \Upsilon^{2}\right) \xi\right] \\
& \left.+k_{1}^{4} k_{2}\left[(12+6 \Upsilon) \xi^{3}+\left(-34 \Upsilon+36 \Upsilon^{2}\right) \xi^{2}+\left(36 \Upsilon^{2}-16 \Upsilon^{3}\right) \xi-16 \Upsilon^{3}\right]\right\} \mid .
\end{aligned}
$$

Further, by suitably arranging the terms yield 


$$
\begin{aligned}
\left|T_{2}(3)\right| & =\mid \frac{\xi^{2}}{2304}\left\{64 k_{3}^{2}-144 k_{2}^{2}+k_{1}^{6}\left[(1-3 \Upsilon) \xi^{4}+\left(-3 \Upsilon+11 \Upsilon^{2}\right) \xi^{3}+\left(2 \Upsilon^{2}-12 \Upsilon^{3}\right) \xi^{2}+4 \Upsilon^{4} \xi\right]\right. \\
& +k_{1}^{2}(-144 \xi+144 \Upsilon)\left[k_{2}-k_{1}^{2}\left(\frac{36 \Upsilon^{2}+36 \xi^{2}-72 \Upsilon \xi}{-144 \xi+144 \Upsilon}\right)\right] \\
& +k_{1} k_{2}(96 \xi-128 \Upsilon)\left[k_{3}-k_{1} k_{2}\left(\frac{-36 \xi^{2}+96 \Upsilon \xi-64 \Upsilon^{2}}{96 \xi-128 \Upsilon}\right)\right] \\
& +k_{1}^{3}\left(16 \Upsilon^{2}+16 \xi^{2}-24 \Upsilon \xi^{2}+16 \Upsilon^{2} \xi-24 \Upsilon \xi\right) \cdot \\
& {\left.\left[k_{3}-k_{1} k_{2}\left(\frac{(-12-6 \Upsilon) \xi^{3}+(-36 \Upsilon+34) \Upsilon \xi^{2}}{16 \Upsilon^{2}+(16-24 \Upsilon) \xi^{2}+\left(16 \Upsilon^{2}-24 \Upsilon\right) \xi}+\frac{(-36+16 \Upsilon) \Upsilon^{2} \xi+16 \Upsilon^{3}+(16-24 \Upsilon) \xi^{2}+\left(16 \Upsilon^{2}-24 \Upsilon\right) \xi}{16}\right)\right]\right\} \mid } \\
& =\mid \frac{\xi^{2}}{2304}\left\{64 k_{3}^{2}-144 k_{2}^{2}+k_{1}^{6}\left[(1-3 \Upsilon) \xi^{4}+\left(-3 \Upsilon+11 \Upsilon^{2}\right) \xi^{3}+\left(2 \Upsilon^{2}-12 \Upsilon^{3}\right) \xi^{2}+4 \Upsilon^{4} \xi\right]\right. \\
& +k_{1}^{2}(-144 \xi+144 \Upsilon)\left[k_{2}-\mu k_{1}^{2}\right]+k_{1} k_{2}(96 \xi-128 \Upsilon)\left[k_{3}-\chi k_{1} k_{2}\right] \\
& \left.+k_{1}^{3}\left(16 \Upsilon^{2}+(16-24 \Upsilon) \xi^{2}+\left(16 \Upsilon^{2}-24 \Upsilon\right) \xi\right)\left[k_{3}-\lambda k_{1} k_{2}\right]\right\} \mid
\end{aligned}
$$

where

$$
\begin{aligned}
& \mu=\frac{36 \Upsilon^{2}+36 \xi^{2}-72 \Upsilon \xi}{-144 \xi+144 \Upsilon} \\
& \chi=\frac{-36 \xi^{2}+96 \Upsilon \xi-64 \Upsilon^{2}}{96 \xi-128 \Upsilon}
\end{aligned}
$$

and

$$
\lambda=\frac{-12 \xi^{3}-6 \Upsilon \xi^{3}-36 \Upsilon^{2} \xi^{2}+34 \Upsilon \xi^{2}-36 \Upsilon^{2} \xi+16 \Upsilon^{3} \xi+16 \Upsilon^{3}}{16 \Upsilon^{2}+16 \xi^{2}-24 \Upsilon \xi^{2}+16 \Upsilon^{2} \xi-24 \Upsilon \xi} .
$$

Consequently, by the triangle inequality, from (3.9), we get

$$
\begin{aligned}
\left|T_{2}(3)\right| \leq & \frac{T^{2}}{2304}\left\{64\left|k_{3}\right|^{2}+144\left|k_{2}\right|^{2}+\left|k_{1}\right|^{6}\left|(1-3 \Upsilon) \xi^{4}+\left(-3 \Upsilon+11 \Upsilon^{2}\right) \xi^{3}+\left(-12 \Upsilon^{3}+2 \Upsilon^{2}\right) \xi^{2}+4 \Upsilon^{4} \xi\right|\right. \\
& +\left|k_{1}\right|^{2}|-144 \xi+144 \Upsilon|\left|k_{2}-\mu k_{1}^{2}\right|+\left|k_{1}\right|\left|k_{2}\right||96 \xi-128 \Upsilon|\left|k_{3}-\chi k_{1} k_{2}\right| \\
& \left.+\left|k_{1}\right|^{3}\left|16 \Upsilon^{2}+(16-24 \Upsilon) \xi^{2}+\left(16 \Upsilon^{2}-24 \Upsilon\right) \xi\right|\left|k_{3}-\lambda k_{1} k_{2}\right|\right\} .
\end{aligned}
$$

By Lemma 2.2, 


$$
\begin{aligned}
\left|k_{2}-\mu k_{1}^{2}\right| & \leq 2 \max \{1,|2 \mu-1|\} \\
& =2 \max \left\{1,\left|\frac{72 \Upsilon^{2}-144 \Upsilon+72 \xi^{2}-144 \Upsilon \xi+144 \xi}{-144 \xi+144 \Upsilon}\right|\right\}, \\
\left|k_{3}-\chi k_{1} k_{2}\right| & \leq 2 \max \{1,|2 \chi-1|\} \\
& =2 \max \left\{1,\left|\frac{-128 \Upsilon^{2}+128 \Upsilon-72 \xi^{2}+192 \Upsilon \xi-96 \xi}{96 \xi-128 \Upsilon}\right|\right\}
\end{aligned}
$$

and

$$
\begin{aligned}
\left|k_{3}-\lambda k_{1} k_{2}\right| & \leq 2 \max \{1,|2 \lambda-1|\} \\
& =2 \max \left\{1, \mid \frac{-16 \Upsilon^{2}+32 \Upsilon^{3}+\left(24 \Upsilon-88 \Upsilon^{2}+32 \Upsilon^{3}\right) \xi+(-24-12 \Upsilon) \xi^{3}}{16 \Upsilon^{2}+16 \xi^{2}-24 \Upsilon \xi^{2}+16 \Upsilon^{2} \xi-24 \Upsilon \xi}\right. \\
& \left.+\frac{\left(-16+92 \Upsilon-72 \Upsilon^{2}\right) \xi^{2}}{16 \Upsilon^{2}+16 \xi^{2}-24 \Upsilon \xi^{2}+16 \Upsilon^{2} \xi-24 \Upsilon \xi} \mid\right\} .
\end{aligned}
$$

By making use of Lemma 2.1 together with (3.11)-(3.13), we find that

$$
\begin{gathered}
\left|k_{1}\right|^{2}|-144 \xi+144 \Upsilon|\left|k_{2}-\mu k_{1}^{2}\right| \leq 8|-144 \xi+144 \Upsilon|\left|\frac{72 \Upsilon^{2}-144 \Upsilon+72 \xi^{2}+(-144 \Upsilon+144) \xi}{-144 \xi+144 \Upsilon}\right|, \\
\left|k_{1}\right|\left|k_{2}\right||96 \xi-128 \Upsilon|\left|k_{3}-\chi k_{1} k_{2}\right| \leq 8|96 \xi-128 \Upsilon|\left|\frac{-128 \Upsilon^{2}+128 \Upsilon-72 \xi^{2}+(192 \Upsilon-96) \xi}{96 \xi-128 \Upsilon}\right|
\end{gathered}
$$

and

$$
\begin{aligned}
& \left|k_{1}\right|^{3}\left|16 \Upsilon^{2}+(16-24 \Upsilon) \xi^{2}+\left(16 \Upsilon^{2}-24 \Upsilon\right) \xi\right|\left|k_{3}-\lambda k_{1} k_{2}\right| \\
& \leq 16\left|16 \Upsilon^{2}+(16-24 \Upsilon) \xi^{2}+\left(16 \Upsilon^{2}-24 \Upsilon\right) \xi\right| \mid \frac{-16 \Upsilon^{2}+32 \Upsilon^{3}+\left(24 \Upsilon-88 \Upsilon^{2}+32 \Upsilon^{3}\right) \xi}{16 \Upsilon^{2}+(16-24 \Upsilon) \xi^{2}+\left(16 \Upsilon^{2}-24 \Upsilon\right) \xi} \\
& +\frac{(-24-12 \Upsilon) \xi^{3}+\left(-16+92 \Upsilon-72 \Upsilon^{2}\right) \xi^{2}}{16 \Upsilon^{2}+(16-24 \Upsilon) \xi^{2}+\left(16 \Upsilon^{2}-24 \Upsilon\right) \xi} \mid
\end{aligned}
$$

Again by applying Lemma 2.1 along with (3.14)-(3.16), from (3.10), we obtain 


$$
\begin{aligned}
\left|T_{2}(3)\right| \leq & \frac{T^{2}}{2304}\left\{832+64\left|(1-3 \Upsilon) \xi^{4}+\left(-3 \Upsilon+11 \Upsilon^{2}\right) \xi^{3}+\left(-12 \Upsilon^{3}+2 \Upsilon^{2}\right) \xi^{2}+4 \Upsilon^{4} \xi\right|\right. \\
& +8\left|72 \Upsilon^{2}-144 \Upsilon+72 \xi^{2}+(-144 \Upsilon+144) \xi\right|+8\left|-128 \Upsilon^{2}+128 \Upsilon-72 \xi^{2}+(192 \Upsilon-96) \xi\right| \\
& \left.+16\left|-16 \Upsilon^{2}+32 \Upsilon^{3}+\left(24 \Upsilon-88 \Upsilon^{2}+32 \Upsilon^{3}\right) \xi+(-24-12 \Upsilon) \xi^{3}+\left(-16+92 \Upsilon-72 \Upsilon^{2}\right) \xi^{2}\right|\right\} .
\end{aligned}
$$

The result is sharp for the function given by $\left\{e^{i \alpha} \frac{z f^{\prime}(z)}{g(z)}-\delta-i \sin \alpha\right\} \frac{1}{t_{\alpha \delta}}=\frac{1+z}{1-z}$. This completes the proof of Theorem 3.1.

Remark 3.1. For $\alpha=0, \delta=0, A=1$ and $B=-1$, Theorem 3.1 yields $\left|T_{2}(3)\right| \leq 25$. This inequality coincides with the result obtained by Ali et al. [14] for $S^{*}$.

Theorem 3.2. If the function $f(z)$ given by (1.1) belongs to the class $S_{C}{ }^{*}(\alpha, \delta, A, B)$, then

$$
\begin{aligned}
\left|T_{3}(3)\right| \leq & \frac{T}{384}\left\{8\left|-12 \Upsilon^{3}+36 \Upsilon^{2}+\left(22 \Upsilon^{2}-44 \Upsilon\right) \xi+(-12 \Upsilon+12) \xi^{2}+2 \xi^{3}\right|\right. \\
& +16|12 \Upsilon-6-8 \xi|+4|-48 \Upsilon+48 \xi|+4|24 \Upsilon-12 \xi|+192\} \cdot \\
& \frac{T^{2}}{9216}\left\{2304+2048+8|-144 \Upsilon+144 \xi|+8\left|-288 \Upsilon^{2}+576 \Upsilon-288 \xi^{2}+(576 \Upsilon-576) \xi\right|\right. \\
& +64\left|(24 \Upsilon-5) \xi^{4}+\left(3 \Upsilon-88 \Upsilon^{2}\right) \xi^{3}+\left(35 \Upsilon^{2}+96 \Upsilon^{3}\right) \xi^{2}+\left(-51 \Upsilon^{3}-32 \Upsilon^{4}\right) \xi+18 \Upsilon^{4}\right| \\
& +4|-144 \xi+288 \Upsilon-288|+8\left|360 \xi^{2}+(-792 \Upsilon+576) \xi+448 \Upsilon^{2}-736 \Upsilon\right| \\
& \left.+16\left|(108+96 \Upsilon) \xi^{3}+\left(32-328 \Upsilon+576 \Upsilon^{2}\right) \xi^{2}+\left(48 \Upsilon+92 \Upsilon^{2}+32 \Upsilon^{3}\right) \xi-256 \Upsilon^{3}-16 \Upsilon^{2}\right|\right\}
\end{aligned}
$$

where $\xi=T e^{-i \alpha}, T=(A-B) t_{\alpha \delta}, t_{\alpha \delta}=\cos \alpha-\delta$ and $\Upsilon=1+B$

Proof. Upon simplification of (1.9), the determinant $T_{3}(3)$ can be written as

$$
\left|T_{3}(3)\right|=\left|\left(a_{3}-a_{5}\right)\left(a_{3}^{2}-2 a_{4}^{2}+a_{3} a_{5}\right)\right|
$$

and by using the triangle inequality, we get

$$
\left|T_{3}(3)\right| \leq\left|a_{3}-a_{5}\right|\left|a_{3}^{2}-2 a_{4}^{2}+a_{3} a_{5}\right| .
$$

Now, equating the coefficient of $z^{5}$ in the expansion of (3.4) and for simplicity, we take $\xi=T e^{-i \alpha}$ and $\Upsilon=1+B$, give us

$$
\begin{aligned}
a_{5}= & \frac{48 k_{4} \xi+32 k_{1} k_{3} \xi^{2}-48 k_{1} k_{3} \Upsilon \xi+12 k_{2}{ }^{2} \xi^{2}-24 k_{2}{ }^{2} \Upsilon \xi+k_{1}{ }^{4} \xi^{4}-6 k_{1}^{4} \Upsilon \xi^{3}}{384} \\
& +\frac{11 k_{1}^{4} \Upsilon^{2} \xi^{2}-6 k_{1}{ }^{4} \Upsilon^{3} \xi+12 k_{1}{ }^{2} k_{2} \xi^{3}-44 k_{1}{ }^{2} k_{2} \Upsilon \xi^{2}+36 k_{1}{ }^{2} k_{2} \Upsilon^{2} \xi}{384} .
\end{aligned}
$$


From the equations (3.5) and (3.17), we obtain

$$
\begin{aligned}
\left|a_{3}-a_{5}\right|= & \mid \frac{1}{384}\left\{\left[96 k_{2} \xi+48 k_{1}^{2} \xi^{2}-48 k_{1}^{2} \Upsilon \xi\right]-\left[48 k_{4} \xi+32 k_{1} k_{3} \xi^{2}-48 k_{1} k_{3} \Upsilon \xi+12 k_{2}{ }^{2} \xi^{2}-24 k_{2}{ }^{2} \Upsilon \xi+k_{1}{ }^{4} \xi^{4}\right.\right. \\
& \left.\left.-6 k_{1}^{4} \Upsilon \xi^{3}+11 k_{1}^{4} \Upsilon^{2} \xi^{2}-6 k_{1}^{4} \Upsilon^{3} \xi+12 k_{1}^{2} k_{2} \xi^{3}-44 k_{1}{ }^{2} k_{2} \Upsilon \xi^{2}+36 k_{1}{ }^{2} k_{2} \Upsilon^{2} \xi\right]\right\} \mid \\
= & \frac{1}{384} \mid k_{1}{ }^{4} \xi\left[6 \Upsilon^{3}-11 \Upsilon^{2} \xi+6 \Upsilon \xi^{2}-\xi^{3}\right]+k_{1}{ }^{2} k_{2} \xi\left[-36 \Upsilon^{2}+44 \Upsilon \xi-12 \xi^{2}\right] \\
& +k_{1}{ }^{2} \xi[-48 \Upsilon+48 \xi]+k_{2}^{2} \xi[24 \Upsilon-12 \xi]+k_{1} k_{3} \xi[48 \Upsilon-32 \xi]+96 k_{2} \xi-48 k_{4} \xi \mid .
\end{aligned}
$$

Further, by suitably arranging the terms, we get

$$
\begin{aligned}
\left|a_{3}-a_{5}\right| & =\frac{1}{384} \mid k_{1}^{2} \xi\left[k_{2}\left(-36 \Upsilon^{2}+44 \Upsilon \xi-12 \xi^{2}\right)-k_{1}^{2}\left(-6 \Upsilon^{3}+11 \Upsilon^{2} \xi-6 \Upsilon \xi^{2}+\xi^{3}\right)\right] \\
& +k_{1}^{2} \xi[-48 \Upsilon+48 \xi]+k_{2}^{2} \xi[24 \Upsilon-12 \xi]-8 \xi\left[6 k_{4}-k_{1} k_{3}(6 \Upsilon-4 \xi)\right]+96 k_{2} \xi \mid \\
& =\frac{|\xi|}{384} \mid k_{1}^{2}\left(-36 \Upsilon^{2}+44 \Upsilon \xi-12 \xi^{2}\right)\left[k_{2}-k_{1}^{2}\left(\frac{-6 \Upsilon^{3}+11 \Upsilon^{2} \xi-6 \Upsilon \xi^{2}+\xi^{3}}{-36 \Upsilon^{2}+44 \Upsilon \xi-12 \xi^{2}}\right)\right] \\
& -48\left[k_{4}-k_{1} k_{3}\left(\frac{6 \Upsilon-4 \xi}{6}\right)\right]+k_{1}^{2}[-48 \Upsilon+48 \xi]+k_{2}^{2}[24 \Upsilon-12 \xi]+96 k_{2} \mid \\
& =\frac{|\xi|}{384} \mid k_{1}^{2}\left(-36 \Upsilon^{2}+44 \Upsilon \xi-12 \xi^{2}\right)\left[k_{2}-\chi k_{1}^{2}\right]-48\left[k_{4}-\mu k_{1} k_{3}\right] \\
& +k_{1}^{2}[-48 \Upsilon+48 \xi]+k_{2}^{2}[24 \Upsilon-12 \xi]+96 k_{2} \mid
\end{aligned}
$$

where

$$
\chi=\frac{-6 \Upsilon^{3}+11 \Upsilon^{2} \xi-6 \Upsilon \xi^{2}+\xi^{3}}{-36 \Upsilon^{2}+44 \Upsilon \xi-12 \xi^{2}}
$$

and

$$
\mu=\frac{6 \Upsilon-4 \xi}{6} .
$$

Consequently, by the triangle inequality, from (3.19), we get

$$
\begin{aligned}
\left|a_{3}-a_{5}\right| \leq & \frac{T}{384}\left\{\left|k_{1}\right|^{2}\left|-36 \Upsilon^{2}+44 \Upsilon \xi-12 \xi^{2}\right|\left|k_{2}-\chi k_{1}^{2}\right|+48\left|k_{4}-\mu k_{1} k_{3}\right|\right. \\
& \left.+96\left|k_{2}\right|+\left|k_{1}\right|^{2}|-48 \Upsilon+48 \xi|+\left|k_{2}\right|^{2}|24 \Upsilon-12 \xi|\right\} .
\end{aligned}
$$

By making use of Lemma 2.1 and Lemma 2.2, we find that 


$$
\begin{aligned}
\left|k_{1}\right|^{2}\left|-36 \Upsilon^{2}+44 \Upsilon \xi-12 \xi^{2}\right|\left|k_{2}-\chi k_{1}^{2}\right| & \leq 8\left|-36 \Upsilon^{2}+44 \Upsilon \xi-12 \xi^{2}\right| \cdot \\
& \left|\frac{-12 \Upsilon^{3}+36 \Upsilon^{2}+\left(22 \Upsilon^{2}-44 \Upsilon\right) \xi+(-12 \Upsilon+12) \xi^{2}+2 \xi^{3}}{-36 \Upsilon^{2}+44 \Upsilon \xi-12 \xi^{2}}\right|
\end{aligned}
$$

and

$$
48\left|k_{4}-\mu k_{1} k_{3}\right| \leq 96\left|\frac{12 \Upsilon-6-8 \xi}{6}\right| .
$$

Again by applying Lemma 2.1 along with (3.21) and (3.22), from (3.20) yields

$$
\begin{aligned}
\left|a_{3}-a_{5}\right| \leq & \frac{T}{384}\left\{8\left|-12 \Upsilon^{3}+36 \Upsilon^{2}+\left(22 \Upsilon^{2}-44 \Upsilon\right) \xi+(-12 \Upsilon+12) \xi^{2}+2 \xi^{3}\right|\right. \\
& +16|12 \Upsilon-6-8 \xi|+4|-48 \Upsilon+48 \xi|+4|24 \Upsilon-12 \xi|+192\} .
\end{aligned}
$$

In view of (3.5), (3.7), (3.8), and (3.17), we have

$$
\begin{aligned}
& \left|a_{3}^{2}-2 a_{4}^{2}+a_{3} a_{5}\right| \\
& =\mid \frac{\xi^{2}}{9216}\left\{576 k_{2}^{2}+k_{1}^{4}\left[144 \Upsilon^{2}+144 \xi^{2}-288 \Upsilon \xi\right]+k_{1}^{2} k_{2}[-576 \Upsilon+576 \xi]\right\} \\
& +\frac{\xi^{2}}{9216}\left\{-512 k_{3}^{2}+k_{1}^{6}\left[-8 \xi^{4}+24 \Upsilon \xi^{3}-16 \Upsilon^{2} \xi^{2}+24 \Upsilon \xi^{4}-88 \Upsilon^{2} \xi^{3}+96 \Upsilon^{3} \xi^{2}-32 \Upsilon^{4} \xi\right]\right. \\
& +k_{1} k_{2} k_{3}[-768 \xi+1024 \Upsilon]+k_{1}^{3} k_{3}\left[-128 \xi^{2}+192 \Upsilon \xi-128 \Upsilon^{2}+192 \Upsilon \xi^{2}-128 \Upsilon^{2} \xi\right] \\
& +k_{1}^{2} k_{2}^{2}\left[-288 \xi^{2}+768 \Upsilon \xi-512 \Upsilon^{2}\right]+k_{1}^{4} k_{2}\left[-96 \xi^{3}+272 \Upsilon \xi^{2}-288 \Upsilon^{2} \xi+128 \Upsilon^{3}\right. \\
& \left.\left.-48 \Upsilon \xi^{3}-288 \Upsilon^{2} \xi^{2}+128 \Upsilon^{3} \xi\right]\right\}+\frac{\xi^{2}}{9216}\left\{288 k_{2} k_{4}+k_{2}^{3}[72 \xi-144 \Upsilon]+k_{1} k_{2} k_{3}[192 \xi-288 \Upsilon]\right. \\
& +k_{1}^{6}\left[3 \xi^{4}-21 \Upsilon \xi^{3}+51 \Upsilon^{2} \xi^{2}-51 \Upsilon^{3} \xi+18 \Upsilon^{4}\right]+k_{1}^{3} k_{3}\left[96 \xi^{2}-240 \Upsilon \xi+144 \Upsilon^{2}\right] \\
& +k_{1}^{2} k_{4}[-144 \Upsilon+144 \xi]+k_{1}^{2} k_{2}^{2}\left[108 \xi^{2}-372 \Upsilon \xi+288 \Upsilon^{2}\right] \\
& \left.+k_{1}^{4} k_{2}\left[42 \xi^{3}-204 \Upsilon \xi^{2}+306 \Upsilon^{2} \xi-144 \Upsilon^{3} \xi\right]\right\} \mid \\
& =\frac{\left|\xi^{2}\right|}{9216} \mid\left\{576 k_{2}^{2}-512 k_{3}^{2}+k_{1}^{2} k_{4}[-144 \Upsilon+144 \xi]\right. \\
& +k_{1}^{6}\left[(24 \Upsilon-5) \xi^{4}+\left(3 \Upsilon-88 \Upsilon^{2}\right) \xi^{3}+\left(35 \Upsilon^{2}+96 \Upsilon^{3}\right) \xi^{2}+\left(-32 \Upsilon^{4}-51 \Upsilon^{3}\right) \xi+18 \Upsilon^{4}\right] \\
& +k_{1}^{2} k_{2}[-576 \Upsilon+576 \xi]+k_{1}^{4}\left[144 \Upsilon^{2}+144 \xi^{2}-288 \Upsilon \xi\right]+288 k_{2} k_{4}+k_{2}^{3}[72 \xi-144 \Upsilon] \\
& +k_{1} k_{2} k_{3}[-576 \xi+736 \Upsilon]+k_{1}^{2} k_{2}^{2}\left[-180 \xi^{2}+396 \Upsilon \xi-224 \Upsilon^{2}\right] \\
& +k_{1}^{3} k_{3}\left[(-32+192 \Upsilon) \xi^{2}+\left(-48 \Upsilon-128 \Upsilon^{2}\right) \xi+16 \Upsilon^{2}\right] \\
& \left.+k_{1}^{4} k_{2}\left[(-54-48 \Upsilon) \xi^{3}+\left(68 \Upsilon-288 \Upsilon^{2}\right) \xi^{2}+\left(18 \Upsilon^{2}-16 \Upsilon^{3}\right) \xi+128 \Upsilon^{3}\right]\right\} \mid \cdot
\end{aligned}
$$


By suitably arranging the terms, we get

$$
\begin{aligned}
& \left|a_{3}^{2}-2 a_{4}^{2}+a_{3} a_{5}\right| \\
& =\frac{\left|\xi^{2}\right|}{9216} \mid\left\{576 k_{2}^{2}-512 k_{3}^{2}+k_{1}^{2} k_{4}[-144 \Upsilon+144 \xi]\right. \\
& +k_{1}^{6}\left[(24 \Upsilon-5) \xi^{4}+\left(3 \Upsilon-88 \Upsilon^{2}\right) \xi^{3}+\left(35 \Upsilon^{2}+96 \Upsilon^{3}\right) \xi^{2}+\left(-32 \Upsilon^{4}-51 \Upsilon^{3}\right) \xi+18 \Upsilon^{4}\right] \\
& +k_{1}^{2}(-576 \Upsilon+576 \xi)\left[k_{2}-k_{1}^{2}\left(\frac{-144 \Upsilon^{2}-144 \xi^{2}+288 \Upsilon \xi}{-576 \Upsilon+576 \xi}\right)\right]+288 k_{2}\left[k_{4}-k_{2}^{2}\left(\frac{-72 \xi+144 \Upsilon}{288}\right)\right] \\
& +k_{1} k_{2}(-576 \xi+736 \Upsilon)\left[k_{3}-k_{1} k_{2}\left(\frac{180 \xi^{2}-396 \Upsilon \xi+224 \Upsilon^{2}}{-576 \xi+736 \Upsilon}\right)\right] \\
& +k_{1}^{3}\left[(-32+192 \Upsilon) \xi^{2}+\left(-48 \Upsilon-128 \Upsilon^{2}\right) \xi+16 \Upsilon^{2}\right] \cdot \\
& \left.\left[k_{3}-k_{1} k_{2}\left(\frac{(54+48 \Upsilon) \xi^{3}+\left(-68 \Upsilon+288 \Upsilon^{2}\right) \xi^{2}+\left(-18 \Upsilon^{2}+16 \Upsilon^{3}\right) \xi-128 \Upsilon^{3}}{(-32+192 \Upsilon) \xi^{2}+\left(-48 \Upsilon-128 \Upsilon^{2}\right) \xi+16 \Upsilon^{2}}\right)\right]\right\} \mid
\end{aligned}
$$

and further yields

$$
\begin{aligned}
& \left|a_{3}^{2}-2 a_{4}^{2}+a_{3} a_{5}\right| \\
& =\frac{\left|\xi^{2}\right|}{9216} \mid\left\{576 k_{2}^{2}-512 k_{3}^{2}+k_{1}^{2} k_{4}[-144 \Upsilon+144 \xi]\right. \\
& +k_{1}^{6}\left[(24 \Upsilon-5) \xi^{4}+\left(3 \Upsilon-88 \Upsilon^{2}\right) \xi^{3}+\left(35 \Upsilon^{2}+96 \Upsilon^{3}\right) \xi^{2}+\left(-32 \Upsilon^{4}-51 \Upsilon^{3}\right) \xi+18 \Upsilon^{4}\right] \\
& +k_{1}^{2}(-576 \Upsilon+576 \xi)\left[k_{2}-\gamma k_{1}^{2}\right]+288 k_{2}\left[k_{4}-\eta k_{2}^{2}\right]+k_{1} k_{2}(-576 \xi+736 \Upsilon)\left[k_{3}-v k_{1} k_{2}\right] \\
& \left.+k_{1}^{3}\left[(-32+192 \Upsilon) \xi^{2}+\left(-48 \Upsilon-128 \Upsilon^{2}\right) \xi+16 \Upsilon^{2}\right]\left[k_{3}-\lambda k_{1} k_{2}\right]\right\} \mid
\end{aligned}
$$

where

$$
\begin{gathered}
\gamma=\frac{-144 \Upsilon^{2}-144 \xi^{2}+288 \Upsilon \xi}{-576 \Upsilon+576 \xi}, \\
\eta=\frac{-72 \xi+144 \Upsilon}{288}, \\
\nu=\frac{180 \xi^{2}-396 \Upsilon \xi+224 \Upsilon^{2}}{-576 \xi+736 \Upsilon}
\end{gathered}
$$


and

$$
\lambda=\frac{(54+48 \Upsilon) \xi^{3}+\left(-68 \Upsilon+288 \Upsilon^{2}\right) \xi^{2}+\left(-18 \Upsilon^{2}+16 \Upsilon^{3}\right) \xi-128 \Upsilon^{3}}{(-32+192 \Upsilon) \xi^{2}+\left(-48 \Upsilon-128 \Upsilon^{2}\right) \xi+16 \Upsilon^{2}} .
$$

Consequently, by the triangle inequality, from (3.24), we obtain

$$
\begin{aligned}
& \left|a_{3}^{2}-2 a_{4}^{2}+a_{3} a_{5}\right| \\
& \leq \frac{T^{2}}{9216}\left\{576\left|k_{2}\right|^{2}+512\left|k_{3}\right|^{2}+\left|k_{1}\right|^{2}\left|k_{4}\right||-144 \Upsilon+144 \xi|\right. \\
& +\left|k_{1}\right|^{6}\left|(24 \Upsilon-5) \xi^{4}+\left(3 \Upsilon-88 \Upsilon^{2}\right) \xi^{3}+\left(35 \Upsilon^{2}+96 \Upsilon^{3}\right) \xi^{2}+\left(-32 \Upsilon^{4}-51 \Upsilon^{3}\right) \xi+18 \Upsilon^{4}\right| \\
& +\left|k_{1}\right|^{2}|-576 \Upsilon+576 \xi|\left|k_{2}-\gamma k_{1}^{2}\right|+288\left|k_{2}\right|\left|k_{4}-\eta k_{2}^{2}\right|+\left|k_{1}\right|\left|k_{2}\right||-576 \xi+736 \Upsilon|\left|k_{3}-v k_{1} k_{2}\right| \\
& \left.+\left|k_{1}\right|^{3}\left|(-32+192 \Upsilon) \xi^{2}+\left(-48 \Upsilon-128 \Upsilon^{2}\right) \xi+16 \Upsilon^{2}\right|\left|k_{3}-\lambda k_{1} k_{2}\right|\right\} .
\end{aligned}
$$

(3.25)

By Lemma 2.2,

$$
\begin{aligned}
\left|k_{2}-\gamma k_{1}^{2}\right| & \leq 2 \max \{1,|2 \gamma-1|\} \\
& =2 \max \left\{1,\left|\frac{-288 \Upsilon^{2}+576 \Upsilon-288 \xi^{2}+(576 \Upsilon-576) \xi}{-576 \Upsilon+576 \xi}\right|\right\}, \\
\left|k_{4}-\eta k_{2}^{2}\right| & \leq 2 \max \{1,|2 \eta-1|\} \\
& =2 \max \left\{1, \frac{|-144 \xi+288 \Upsilon-288|}{288}\right\}, \\
\left|k_{3}-v k_{1} k_{2}\right| & \leq 2 \max \{1,|2 v-1|\} \\
& =2 \max \left\{1, \mid \frac{360 \xi^{2}+(-792 \Upsilon+576) \xi+448 \Upsilon^{2}-736 \Upsilon}{-576 \xi+736 \Upsilon}\right\}
\end{aligned}
$$

and

$$
\begin{aligned}
\left|k_{3}-\lambda k_{1} k_{2}\right| & \leq 2 \max \{1,|2 \lambda-1|\} \\
& =2 \max \left\{1,\left|\frac{(108+96 \Upsilon) \xi^{3}+\left(-328 \Upsilon+576 \Upsilon^{2}+32\right) \xi^{2}+\left(92 \Upsilon^{2}+32 \Upsilon^{3}+48 \Upsilon\right) \xi-256 \Upsilon^{3}-16 \Upsilon^{2}}{(-32+192 \Upsilon) \xi^{2}+\left(-48 \Upsilon-128 \Upsilon^{2}\right) \xi+16 \Upsilon^{2}}\right|\right\} .
\end{aligned}
$$

Hence, applying Lemma 2.1 together with (3.26)-(3.29), we find that 
(3.30)

$$
\begin{gathered}
\left|k_{1}\right|^{2}|-576 \Upsilon+576 \xi|\left|k_{2}-\gamma k_{1}^{2}\right| \leq 8|-576 \Upsilon+576 \xi|\left|\frac{-288 \Upsilon^{2}+576 \Upsilon-288 \xi^{2}+(576 \Upsilon-576) \xi}{-576 \Upsilon+576 \xi}\right|, \\
288\left|k_{2}\right|\left|k_{4}-\eta k_{2}^{2}\right| \leq \frac{1152|-144 \xi+288 \Upsilon-288|}{288}
\end{gathered}
$$

$$
\left|k_{1}\right|\left|k_{2}\right||-576 \xi+736 \Upsilon|\left|k_{3}-v k_{1} k_{2}\right| \leq 8|-576 \xi+736 \Upsilon|\left|\frac{360 \xi^{2}+(-792 \Upsilon+576) \xi+448 \Upsilon^{2}-736 \Upsilon}{-576 \xi+736 \Upsilon}\right|
$$

and

$$
\begin{aligned}
& \left|k_{1}\right|^{3}\left|(-32+192 \Upsilon) \xi^{2}+\left(-48 \Upsilon-128 \Upsilon^{2}\right) \xi+16 \Upsilon^{2}\right|\left|k_{3}-\lambda k_{1} k_{2}\right| \\
& \leq 16\left|(-32+192 \Upsilon) \xi^{2}+\left(-48 \Upsilon-128 \Upsilon^{2}\right) \xi+16 \Upsilon^{2}\right| \\
& \left|\frac{(108+96 \Upsilon) \xi^{3}+\left(-328 \Upsilon+576 \Upsilon^{2}+32\right) \xi^{2}+\left(92 \Upsilon^{2}+32 \Upsilon^{3}+48 \Upsilon\right) \xi-256 \Upsilon^{3}-16 \Upsilon^{2}}{(-32+192 \Upsilon) \xi^{2}+\left(-48 \Upsilon-128 \Upsilon^{2}\right) \xi+16 \Upsilon^{2}}\right|
\end{aligned}
$$

Again by applying Lemma 2.1 along with (3.30)-(3.33), from (3.25) yields

$$
\begin{aligned}
& \left|a_{3}^{2}-2 a_{4}^{2}+a_{3} a_{5}\right| \\
& \leq \frac{T^{2}}{9216}\left\{2304+2048+8|-144 \Upsilon+144 \xi|+8\left|-288 \Upsilon^{2}+576 \Upsilon-288 \xi^{2}+(576 \Upsilon-576) \xi\right|\right. \\
& +64\left|(24 \Upsilon-5) \xi^{4}+\left(3 \Upsilon-88 \Upsilon^{2}\right) \xi^{3}+\left(35 \Upsilon^{2}+96 \Upsilon^{3}\right) \xi^{2}+\left(-51 \Upsilon^{3}-32 \Upsilon^{4}\right) \xi+18 \Upsilon^{4}\right| \\
& +4|-144 \xi+288 \Upsilon-288|+8\left|360 \xi^{2}+(-792 \Upsilon+576) \xi+448 \Upsilon^{2}-736 \Upsilon\right| \\
& \left.+16\left|(108+96 \Upsilon) \xi^{3}+\left(32-328 \Upsilon+576 \Upsilon^{2}\right) \xi^{2}+\left(48 \Upsilon+92 \Upsilon^{2}+32 \Upsilon^{3}\right) \xi-256 \Upsilon^{3}-16 \Upsilon^{2}\right|\right\} .
\end{aligned}
$$

Finally, from (3.23) and (3.34), we obtain

$$
\begin{aligned}
\left|T_{3}(3)\right| \leq & \frac{T}{384}\left\{8\left|-12 \Upsilon^{3}+36 \Upsilon^{2}+\left(22 \Upsilon^{2}-44 \Upsilon\right) \xi+(-12 \Upsilon+12) \xi^{2}+2 \xi^{3}\right|\right. \\
& +16|12 \Upsilon-6-8 \xi|+4|-48 \Upsilon+48 \xi|+4|24 \Upsilon-12 \xi|+192\} \cdot \\
& \frac{T^{2}}{9216}\left\{2304+2048+8|-144 \Upsilon+144 \xi|+8\left|-288 \Upsilon^{2}+576 \Upsilon-288 \xi^{2}+(576 \Upsilon-576) \xi\right|\right. \\
& +64\left|(24 \Upsilon-5) \xi^{4}+\left(3 \Upsilon-88 \Upsilon^{2}\right) \xi^{3}+\left(35 \Upsilon^{2}+96 \Upsilon^{3}\right) \xi^{2}+\left(-51 \Upsilon^{3}-32 \Upsilon^{4}\right) \xi+18 \Upsilon^{4}\right| \\
& +4|-144 \xi+288 \Upsilon-288|+8\left|360 \xi^{2}+(-792 \Upsilon+576) \xi+448 \Upsilon^{2}-736 \Upsilon\right| \\
& \left.+16\left|(108+96 \Upsilon) \xi^{3}+\left(32-328 \Upsilon+576 \Upsilon^{2}\right) \xi^{2}+\left(48 \Upsilon+92 \Upsilon^{2}+32 \Upsilon^{3}\right) \xi-256 \Upsilon^{3}-16 \Upsilon^{2}\right|\right\} .
\end{aligned}
$$


This completes the proof of Theorem 3.2.

By putting the specific values for the parameters $\alpha, \delta, A$ and $B$ in Theorem 3.1 and Theorem 3.2, we obtain the coefficient bounds for the Toeplitz determinants for the subclasses introduced by El-Ashwah and Thomas [4], Halim [5], and Dahhar and Janteng [6], respectively as follows.

Corollary 3.1. For $f \in S_{C}^{*}(0,0,1,-1)$, we obtain $\left|T_{2}(3)\right| \leq 25$ and $\left|T_{3}(3)\right| \leq 240$.

Corollary 3.2. For $f \in S_{C}^{*}(0, \delta, 1,-1)$, we obtain

$$
\begin{aligned}
\left|T_{2}(3)\right| \leq & \frac{4(1-\delta)^{2}}{2304}\left\{832+64\left|16(1-\delta)^{4}\right|+8\left|288(1-\delta)^{2}+288(1-\delta)\right|\right. \\
& \left.+8\left|-288(1-\delta)^{2}-192(1-\delta)\right|+16\left|-192(1-\delta)^{3}-64(1-\delta)^{2}\right|\right\}
\end{aligned}
$$

and

$$
\begin{aligned}
\left|T_{3}(3)\right| \leq & \frac{2(1-\delta)}{384}\left\{8\left|48(1-\delta)^{2}+16(1-\delta)^{3}\right|+16|-6-16(1-\delta)|+4|96(1-\delta)|+4|-24(1-\delta)|+192\right\} \\
& \frac{4(1-\delta)^{2}}{9216}\left\{2304+2048+8|288(1-\delta)|+8\left|-1152(1-\delta)^{2}-1152(1-\delta)\right|\right. \\
& +64\left|-80(1-\delta)^{4}\right|+4|-288(1-\delta)-288|+8\left|1440(1-\delta)^{2}+1152(1-\delta)\right| \\
& \left.+16\left|864(1-\delta)^{3}+128(1-\delta)^{2}\right|\right\}
\end{aligned}
$$

Corollary 3.3. For $f \in S_{C}{ }^{*}(0,0, A, B)$, we obtain

$$
\begin{aligned}
\left|T_{2}(3)\right| \leq & \frac{(A-B)^{2}}{2304}\left\{832+64 \mid(1-3 \Upsilon)(A-B)^{4}+\left(-3 \Upsilon+11 \Upsilon^{2}\right)(A-B)^{3}\right. \\
& +\left(-12 \Upsilon^{3}+2 \Upsilon^{2}\right)(A-B)^{2}+4 \Upsilon^{4}(A-B) \mid \\
& +8\left|72 \Upsilon^{2}-144 \Upsilon+72(A-B)^{2}+(-144 \Upsilon+144)(A-B)\right| \\
& +8\left|-128 \Upsilon^{2}+128 \Upsilon-72(A-B)^{2}+(192 \Upsilon-96)(A-B)\right| \\
& +16 \mid-16 \Upsilon^{2}+32 \Upsilon^{3}+\left(24 \Upsilon-88 \Upsilon^{2}+32 \Upsilon^{3}\right)(A-B) \\
& \left.+(-24-12 \Upsilon)(A-B)^{3}+\left(-16+92 \Upsilon-72 \Upsilon^{2}\right)(A-B)^{2} \mid\right\}
\end{aligned}
$$


and

$$
\begin{aligned}
\left|T_{3}(3)\right| \leq & \frac{(A-B)}{384}\left\{8\left|-12 \Upsilon^{3}+36 \Upsilon^{2}+\left(22 \Upsilon^{2}-44 \Upsilon\right)(A-B)+(-12 \Upsilon+12)(A-B)^{2}+2(A-B)^{3}\right|\right. \\
& +16|12 \Upsilon-6-8(A-B)|+4|-48 \Upsilon+48(A-B)|+4|24 \Upsilon-12(A-B)|+192\} \cdot \\
& \frac{(A-B)^{2}}{9216}\{2304+2048+8|-144 \Upsilon+144(A-B)| \\
& +8\left|-288 \Upsilon^{2}+576 \Upsilon-288(A-B)^{2}+(576 \Upsilon-576)(A-B)\right| \\
& +64 \mid(24 \Upsilon-5)(A-B)^{4}+\left(3 \Upsilon-88 \Upsilon^{2}\right)(A-B)^{3}+\left(35 \Upsilon^{2}+96 \Upsilon^{3}\right)(A-B)^{2} \\
& +\left(-51 \Upsilon^{3}-32 \Upsilon^{4}\right)(A-B)+18 \Upsilon^{4}|+4|-144(A-B)+288 \Upsilon-288 \mid \\
& +8\left|360(A-B)^{2}+(-792 \Upsilon+576)(A-B)+448 \Upsilon^{2}-736 \Upsilon\right|+16 \mid(108+96 \Upsilon)(A-B)^{3} \\
& \left.+\left(32-328 \Upsilon+576 \Upsilon^{2}\right)(A-B)^{2}+\left(48 \Upsilon+92 \Upsilon^{2}+32 \Upsilon^{3}\right)(A-B)-256 \Upsilon^{3}-16 \Upsilon^{2} \mid\right\} .
\end{aligned}
$$

It is observed that the result of $\left|T_{2}(3)\right|$ for $S^{*}$ and $S_{C}{ }^{*}$ are shown to be equivalent.

\section{CONCLUSION}

In this paper, we have obtained the coefficient bounds for $\left|T_{2}(3)\right|$ and $\left|T_{3}(3)\right|$ for the subclass of tilted starlike functions with respect to conjugate points of order $\delta, S_{C}^{*}(\alpha, \delta, A, B)$. The results obtained can be reduced to the results for some existing subclasses in the literature by considering specific values for the parameters $\alpha, \delta, A$ and $B$.

Acknowledgements: The authors wish to thank the anonymous referees for their careful reading.

Conflicts of Interest: The authors declare that there are no conflicts of interest regarding the publication of this paper.

\section{REFERENCES}

[1] I. Graham, Geometric function theory in one and higher dimensions, CRC Press, New York, 2003.

[2] S. S. Miller, P. T. Mocanu, Second order differential inequalities in the complex plane, J. Math. Anal. Appl. 65(2) (1978), 289-305.

[3] S. S. Miller, P. T. Mocanu, Differential subordinations and univalent functions, Michigan Math. J. 28(2) (1981), 157-172. 
[4] R. M. El-Ashwah, D. K. Thomas, Some subclasses of close-to-convex functions, J. Ramanujan Math. Soc. 2(1) (1987), 85-100.

[5] S. Halim, Functions starlike with respect to other points, Int. J. Math. Math. Sci. 14(3) (1991), 451-456.

[6] S. A. F. M. Dahhar, A. Janteng, A subclass of starlike functions with respect to conjugate points, Int. Math. Forum, 4(28) (2009), 1373-1377.

[7] N. H. A. A. Wahid, D. Mohamad, S. Cik Soh, On a subclass of tilted starlike functions with respect to conjugate points, Menemui Mat. (Discover. Math.) 37(1) (2015), 1-6.

[8] K. Ye, L. H. Lim, Every matrix is a product of Toeplitz matrices, Found. Comput. Math. 16(3) (2016), 577-598.

[9] D. K. Thomas and S. A. Halim, Toeplitz matrices whose elements are the coefficients of starlike and close-to-convex functions, Bull. Malaysian Math. Sci. Soc. 40(4) (2016), 1781-1790.

[10] V. Radhika, S. Sivasubramanian, G. Murugusundaramoorthy, J. M. Jahangiri, Toeplitz matrices whose elements are the coefficients of functions with bounded boundary rotation, J. Complex Anal. 2016 (2016), Art. ID 4960704.

[11] S. Sivasubramanian, M. Govindaraj, G. Murugusundaramoorthy, Toeplitz matrices whose elements are the coefficients of analytic functions belonging to certain conic domains, Int. J. Pure Appl. Math. 109(10) (2016), 39-49.

[12] C. Ramachandran, D. Kavitha, Toeplitz determinant for some subclasses of analytic functions, Glob. J. Pure Appl. Math. 13(2) (2017), 785-793.

[13] N. Magesh, Ş. Altınkaya, S. Yalçın, Construction of Toeplitz matrices whose elements are the coefficients of univalent functions associated with q-derivative operator, ArXiv:1708.03600 [Math]. (2017).

[14] M. F. Ali, D. K. Thomas, A. Vasudevarao, Toeplitz determinants whose elements are the coefficients of analytic and univalent functions, Bull. Aust. Math. Soc. 97(2) (2018), 253-264.

[15] V. Radhika, J. M. Jahangiri, S. Sivasubramanian, G. Murugusundaramoorthy, Toeplitz matrices whose elements are coefficients of Bazilevič functions, Open Math. 16(1) (2018), 1161-1169.

[16] H. M. Srivastava, Q. Z. Ahmad, N. Khan, B. Khan, Hankel and Toeplitz determinants for a subclass of q-starlike functions associated with a general conic domain, Mathematics, 7(2) (2019), 181.

[17] H. Y. Zhang, R. Srivastava, H. Tang, Third-order Hankel and Toeplitz determinants for starlike functions connected with the sine function, Mathematics, 7(5) (2019), 404.

[18] S. N. Al-Khafaji, A. Al-Fayadh, A. H. Hussain, S. A. Abbas, Toeplitz Determinant whose Its Entries are the Coefficients for Class of Non-Bazilevic Functions, J. Phys.: Conf. Ser. 1660 (2020), 012091.

[19] P. L. Duren, Univalent Functions vol. 259, Springer, New York-Berlin-Heidelberg-Tokyo, 1983.

[20] I. Efraimidis, A generalization of Livingston's coefficient inequalities for functions with positive real part, J. Math. Anal. Appl. 435(1) (2016), 369-379. 